\title{
Genetic manipulation of KNAT7 transcription factor enhances saccharification by reducing recalcitrance in the bioenergy crop Populus
}

\author{
YOGESH AHLAWAT * and Chandrashekhar P Joshi *
}

\begin{abstract}
The precise role of KNAT7 transcription factors (TFs) in the regulation of secondary cell wall (SCW) biosynthesis of poplars has largely remained unclear as our understanding of KNAT7 is continuously evolving. Through the use of a variety of novel strategies, we can understand how genetic modifications in homologous and heterologous KNAT7 gene expression affect SCW development. The resulting changes in wood properties could in turn affect the saccharification efficiency of lignocellulosic biomass in transgenic poplars. We produced poplar KNAT7 (PtKNAT7) overexpression (PtKNAT7$O E$ ) and RNAi construct (PtKNAT7-AS) for the generation of transgenic poplars. Since the overexpression of homologous genes can potentially result in co-suppression, we also overexpressed Arabidopsis KNAT7 (AtKNAT7-OE) in transgenic poplars. In all our Agrobacterium vector constructs, the expression of $K N A T 7$ transgenes was driven under developing xylem-specific promoter, DX15. The PtKNAT7-AS lines showed a $6 \%$ reduction in total lignin content in poplar wood compared to the controls. Syringyl to Guaiacyl lignin $(\mathrm{S} / \mathrm{G})$ ratios were significantly increased in all the three transgenic lines from PtKNAT7-OE and PtKNAT7- $A S$ than controls. All transgenic lines including, PtKNAT7-OE, PtKNAT7-AS 1, and AtKNAT7-OE wood yielded significantly greater glucose release than controls. Antisense KNAT7 transgenic lines were taller and the stem was thicker than control. These results suggest targeted modification of KNAT7 is achievable in poplar with enhanced functional wall properties without compromising plant growth and productivity. Overall, our results demonstrated that developing xylem-specific genetic alteration of KNAT7 TFs affects the lignification process as well as improving the saccharification efficiency, hence, providing a powerful tool for improving bioethanol production from bioenergy poplar plants.
\end{abstract}

Keywords: cell wall, secondary cell wall, saccharification 